\title{
Romeo: A Parametric Model-Checker for Petri Nets with Stopwatches
}

Didier Lime, Olivier H. Roux, Charlotte Seidner, and Louis-Marie Traonouez

\author{
IRCCyN, CNRS UMR 6597, Nantes, France \\ \{Didier.Lime, Olivier-h.Roux, Charlotte.Seidner, \\ Louis-Marie.Traonouez\}@irccyn.ec-nantes.fr
}

\begin{abstract}
Last time we reported on Romeo, analyses with this tool were mostly based on translations to other tools. This new version provides an integrated TCTL model-checker and has gained in expressivity with the addition of parameters. Although there exists other tools to compute the state-space of stopwatch models, Romeo is the first one that performs TCTL model-checking on stopwatch models. Moreover, it is the first tool that performs TCTL model-checking on timed parametric models. Indeed, Romeo now features an efficient model-checking of time Petri nets using the Uppaal DBM Library, the model-checking of stopwatch Petri nets and parametric stopwatch Petri nets using the Parma Polyhedra Library and a graphical editor and simulator of these models. Furthermore, its audience has increased leading to several industrial contracts. This paper reports on these recent developments of Romeo.
\end{abstract}

Keywords: Time Petri nets, model-checking, stopwatches, parameters, TCTL, tool.

\section{Introduction}

Time Petri nets $(T P N s)$ [1] are a classical time extension of Petri nets. They allow an easy representation of real-time systems features such as synchronization and parallelism. State reachability is decidable for bounded TPNs, which is sufficient for virtually all practical purposes.

However, it is also often useful to model actions that can be suspended and later resumed. Several extensions of time Petri nets have been proposed to express the preemptive scheduling of tasks, such as Scheduling-TPNS 2], inhibitor hyperarc TPNs (ITPNs) 3] or Preemptive-TPNs 4]. All these models belong to the class of TPNs extended with stopwatches $\left(S_{w} P N_{S}\right)$ [5]. Reachability and most other properties of interest are however undecidable for ${ }_{w} P N s$, even when bounded [5].

Furthermore, the design of a system often benefits from the use of parameters, e.g. when specifications are not yet completely defined. Parametric TPNS $(P T P N s)$ and parametric $S_{W} P N_{S}(P S w P N s)$ are parametric extensions of TPNs and $S_{w} P N_{s}$ that can be used to perform parametric model-checking [6]. The goal is to synthesize constraints on the parameters which helps the system design. 


\section{Presentation of Romeo}

The Romeo tool 11 (available for Linux, MacOSX and Windows platforms) consists of a graphical user interface (GUI) (written in Tcl/Tk), to edit and simulate TPNs, and a computation module Mercutio (written in $\mathrm{C}++$ ), that performs model-checking and state-space computation.

The two other main tools for the analysis of TPNS and $S_{w P N S}$ are TINA [7] and ORIS 88. TINA has many interesting features, including the computation of a graph preserving CTL (Computation Tree Logic) properties and an off-line full LTL model-checker. ORIS also has several unique features of interest among which the most notable is probably the analysis of time Petri nets with stochastic aspects.

Design and Simulation. The GUI allows Petri nets edition and features three different modes for each supported extension (TPNs, Scheduling-TPNs or $I T P N$ s). In each mode, a parametric extension is available (PTPNs, SchedulingPTPNs or PITPNs). In these latter extensions, ROMEO supports the use of parametric linear expressions in the time bounds of the transitions, and allows to add linear constraints on the parameters to restrict their domain.

The GUI also features an on-line interactive simulator for scenario testing. It allows to study a particular trace in the state-space of the model, either with the state-class method [9] or with the zone-based method [10] (only for TPNs).

On-the-Fly Model-Checking. Through the computation module Mercutio, Romeo can perform model-checking of time Petri nets models for quantitative temporal logic formulae (TCTL) [11. We consider a restricted subset of TCTL formulae with no recursion in the formulae for which we can propose an efficient on-the-fly model-checking. Moreover, this subset appears to be sufficient to verify many interesting properties on time models. Reachability properties can be checked with formulae such as $\exists \diamond_{[a, b]}(p)$ (where $[a, b]$ is a time interval, with $b$ possibly infinite, and $p$ a property on the markings of the net) and safety properties with $\forall \square_{[a, b]}(p)$. Liveness properties can be checked with $\forall \diamond_{[a, b]}(p)$ or by using a bounded response property such as $p \rightsquigarrow[0, b] q$. It is equivalent to $\forall \square\left(p \Rightarrow \forall \diamond_{[0, b]}(q)\right)$, and thus allows one level of recursion.

This subset allows to implement efficient model-checking algorithms for TPNS with the state-class graph [12. In bounded TPNs, the algorithm is based on DBMs (Difference Bounds Matrix) and the implementation uses the Uppaal DBM Library [13] to encode the firing domains. For $S_{w} P N_{s}$ and parametric $\mathrm{SW}_{W} \mathrm{~N}_{S}$, the reachability problem is undecidable and as a consequence semialgorithms are implemented in Romeo. In these models, firing domains are encoded with polyhedra by using the Parma Polyhedra Library [14].

With parametric nets, ROMEO can verify parametric TCTL formulae in which the bounds of the temporal constraints ( $a$ and $b$ in the above examples) can be replaced by parameters. The goal is to determine the valuations of these parameters, such that for these valuations the model verifies the formula. The

${ }^{1}$ Download at: http://romeo.rts-software.org/ 
semi-algorithms implemented are based on the parametric state-class graph [6]. As a result, RoMEO synthesizes a set of constraints (a disjunction of polyhedra, also encoded with the Parma Polyhedra Library) to represent the set of these valuations.

State-Space Computation. Romeo implements two state-space computation methods, the state-class graph and the zone-based graph.

RomeO computes the state-class graph (SCG) that preserves LTL properties [9. The algorithm is based on DBMs for bounded TPNS and the semialgorithm is based on polyhedra for PTPNs. For ${ }_{W} P N_{s}$, exact computation semi-algorithms are implemented by using either polyhedra or both polyhedra and DBMs. Finally an overapproximating semi-algorithm is also available for SwPNs and uses DBMs.

For TPNs, RomEO generates a zone-based graph [10] that preserves markings and converges by inclusion.

Finally, translations from TPNS to Timed Automata are available through the state-class automaton or by a structural translation. $S w P N s$ can be translated into Stopwatch Automata by an overapproximation method. Both were accounted for in [15].

\section{New Functionalities}

Since the last paper about Romeo 15, several enhancements have been made to the tool.

Regarding expressiveness, we have added the support for several classical special arcs: read arcs (resp. logical inhibitor arcs) test the number of tokens in a place, without consuming them, for the relation "greater than" (resp. "less than"). Reset arcs empty a place of all its token (regardless of their number) after the firing of a transition.

Furthermore, in addition to the scheduling extension, stopwatches can now be defined using time inhibitor arcs $[3]$.

Regarding verification, we now have a model-checker for TCTL properties for $T P N_{s}, S_{w P N s}$ and their parametric counterparts. It allows to work with RoMEO without the help of other tools.

This is, to our knowledge, the first TCTL model-checker for (bounded) time Petri nets but also the first one for stopwatch models.

Furthermore, as already mentioned, ROMEO now implements a new framework for the design and verification of parametric time Petri nets as described in [6]. The associated parametric model-checker is also, to our knowledge, the first one to perform TCTL parametric model-checking on timed parametric models.

\section{Conclusion}

Romeo is one of the three main tools on time and stopwatch Petri nets. It performs state-space computation and simulation based on both the state-class 
method and the zone-based method. Moreover, it performs on-the-fly TCTL model-checking of time, stopwatch and parametric Petri nets. Its implementation is very efficient thanks to the use of two robust libraries, namely the Uppaal DBM Library and the Parma Polyhedra Library.

RomEO has many industrial users such as DGA, SODIUS, Dassault Aviation and $E A D S$ leading to several industrial contracts and partnerships.

\section{References}

1. Merlin, P.: A study of the recoverability of computing systems. PhD thesis, Department of Information and Computer Science, Univ. of California, Irvine (1974)

2. Roux, O., Déplanche, A.M.: A t-time Petri net extension for real time-task scheduling modeling. European Journal of Automation (JESA) 36(7), 973-987 (2002)

3. Roux, O.H., Lime, D.: Time petri nets with inhibitor hyperarcs. Formal semantics and state space computation. In: Cortadella, J., Reisig, W. (eds.) ICATPN 2004. LNCS, vol. 3099, pp. 371-390. Springer, Heidelberg (2004)

4. Bucci, G., Fedeli, A., Sassoli, L., Vicario, E.: Time state space analysis of real-time preemptive systems. IEEE trans. on Soft. Eng. 30(2), 97-111 (2004)

5. Berthomieu, B., Lime, D., Roux, O.H., Vernadat, F.: Reachability problems and abstract state spaces for time Petri nets with stopwatches. Discrete Event Dynamic Systems 17(2), 133-158 (2007)

6. Traonouez, L.-M., Lime, D., Roux, O.H.: Parametric model-checking of time Petri nets with stopwatches using the state-class graph. In: Cassez, F., Jard, C. (eds.) FORMATS 2008. LNCS, vol. 5215, pp. 280-294. Springer, Heidelberg (2008)

7. Berthomieu, B., Ribet, P.O., Vernadat, F.: The tool tina - construction of abstract state spaces for Petri nets and time Petri nets. Int. Journal of Production Research 42(4) (July 2004), http://www.laas.fr/tina/

8. Bucci, G., Sassoli, L., Vicario, E.: Oris: A tool for state-space analysis of real-time preemptive systems. In: QEST 2004 (2004)

9. Berthomieu, B., Diaz, M.: Modeling and verification of time dependent systems using time Petri nets. IEEE trans. on Soft. Eng. 17(3), 259-273 (1991)

10. Gardey, G., Roux, O.H., Roux, O.F.: State space computation and analysis of time Petri nets. Theory and Practice of Logic Programming (TPLP) 6(3), 301-320 (2006); Copyright Cambridge Press

11. Alur, R., Courcoubetis, C., Dill, D.: Model-checking in dense real-time. Information and Computation 104, 2-34 (1993)

12. Hadjidj, R., Boucheneb, H.: On-the-fly TCTL model checking for time Petri nets using state class graphs. In: ACSD, pp. 111-122. IEEE Computer Society Press, Los Alamitos (2006)

13. Larsen, K.G., Pettersson, P., Yi, W.: UPPAAL in a nutshell. International Journal on Software Tools for Technology Transfer 1(1-2), 134-152 (1997)

14. Bagnara, R., Hill, P.M., Zaffanella, E.: The Parma Polyhedra Library. Quaderno 457, Dipartimento di Matematica, Università di Parma, Italy (2006)

15. Gardey, G., Lime, D., Magnin, M., Roux, O(H.): Roméo: A tool for analyzing time petri nets. In: Etessami, K., Rajamani, S.K. (eds.) CAV 2005. LNCS, vol. 3576, pp. 418-423. Springer, Heidelberg (2005) 